九州大学学術情報リポジトリ

Kyushu University Institutional Repository

\title{
STOPPED DECISION PROCESS ON COMPACT METRIC SPACES
}

Iwamoto, Sei ichi

Department of Mathematics, Kyushu University

https://doi.org/10.5109/13044

出版情報: 統計数理研究. 14 (1/2)，pp.51-60，1970-03. Research Association of Statistical Sciences

バージョン :

権利関係 : 


\title{
STOPPED DECISION PROCESSES ON COMPACT METRIC SPACES
}

\author{
By
}

\author{
Seiichi IW AMOTo*
}

(Received February 5, 1970)

\section{$\S 1$. Introduction.}

A stopped decision process is specified by six objects; $S, A, q, r, g$ and $\alpha$. S, the set of the possible states of some system, is a non-empty Borel subset of a Polish space and $A$, the set of actions available to you, is a non-empty Borel subset of the Polish space. At each time $n$, suppose that when the system is in $s$ two alternatives are possible (a) action $a$ is chosen or (b) the next sampling is terminated (stopped). The election (a) moves the system to a new state $s^{\prime}$ (which will be the state you observe tomorrow) according to the distribution $q(\cdot \mid s, a)$ and consequently you receive a reward $r\left(s, a, s^{\prime}\right)$, and election (b) terminates the process and you will receive the terminal reward $g(s)$. Since $\alpha(<1)$ is a discount factor, unit reward on the $n$-th day is worth only $\alpha^{n-1}$.

A stopped policy is pair $(\pi, \tau)$. The policy $\pi$ is a sequence $\pi_{1}, \pi_{2}, \cdots$ where $\pi_{n}$ tells you how to select an action on the $n$-th day as a function of the previous history $h=\left(s_{1}, a_{1}, \cdots, a_{n-1}, s_{n}\right)$ of the system, by associating with each $h$ (Borel measurably) a probability distribution $\pi_{n}(\cdot \mid h)$ on the Borel field of $A$. $\tau$ is an essentially finite, non-anticipative integer valued random variable. Then such a $\tau$ defines a stopping rule: If $\tau=n$, then choose (b) and the process is terminated at time $n$; if $\tau>n$ choose (a) at time $n$ and the system goes to $(n+1)$-st stage.

The stopped policy $(\pi, \tau)$ associates with each initial state $s_{1}$ a corresponding $n$-th day return

$$
\sum_{k=1}^{n-1} \alpha^{k-1} r\left(s_{k}, a_{k}, s_{k+1}\right)+\alpha^{n-1} g\left(s_{n}\right)
$$

at $\tau=n$, where for each $k, s_{k}$ is the state of the system, and $a_{k}$ the action at time $k$.

Note that only when you take the stopping rule $\tau=n$, you can receive an income which is the sum of the discounted reward by choosing (a) up to date and the discounted one gained by stopping at $\tau=n$.

The stopped decision problem is to maximize your total expected reward over the future

A Baire function $f$ from $S$ into $A$ defines a policy: when in state $s$ at time $n$, choose an action $f(s)$ independently of when and how you have arrived at state $s$, where $\tau$ is the paired stopping time. Such a policy will be called stationary policy,

* Department of Mathematics, Kyushu University, Fukuoka. 
which is denoted by $f^{\infty}$. A stationary stopped-policy is one whose policy is stationary and whose stopping time is Markov (Section 3).

Recently the stopped decision problem has been studied extensively by N. Furukawa and S. Iwamoto ([2]), and the existence of stationary stopped-policies, which are optimal in some senses, was shown under some assumptions.

In this paper, we will make three additional assumptions about $A, q, r$ and $g$. In Section 4 , the following assumptions will remain operative; (1) $A$ is a compact metric space, (2) $r\left(s, a, s^{\prime}\right)=r^{*}(s, a)+r^{* *}\left(s^{\prime}\right)$, where $r^{*}, r^{* *}$ and $g$ are bounded upper semi-continuous and (3) if $s_{n} \rightarrow s, a_{n} \rightarrow a$, then $q\left(\cdot \mid s_{n}, a_{n}\right)$ converges weakly to $q(\cdot \mid s, a)$. Our result is the following: under these assumptions, there always exists an $\varepsilon$-optimal stationary stopped-policy for any $\varepsilon>0$.

Our method of proof is based on two facts; (A) The optimality equation has at most one bounded solution ([2]), and (B) Selection Theorem, due to Dubins and Savage ([3]), which was proved by A. Maitra in detail.

In Section 2 we give the notations and definitions to be used throughout this paper, and in Section 3 we shall define the stopped decision process. Section 4 is devoted to the existence theorem of $\varepsilon$-optimal stationary stopped policy, preparing the fundamental lemmas.

\section{$\S 2$. Notations and probabilistic definitions.}

This section is devoted to an exposition of the basic notations and probilistic definitions needed for the following sections. We follow [2] as closely as possible.

By a Borel set we mean a Borel subset of some Polish (i. e. complete separable metric) space. By a probability measure on a non-empty Borel set $X$ we mean a probability measure defined on the Borel field of $X$, and the class of all probability measures on $X$ is denoted by $P(X)$. For any non-empty Borel sets $X, Y$, a conditional probability measure on $Y$ given $X$ is denoted by $Q(Y \mid X)$. The class of all bounded Baire functions on $X$ is denoted by $M(X) . C_{1}(X)$ is the class of all bounded upper semi-continuous functions on $X$. It is clear that $C_{1}(X) \leqq M(X)$. If $u, v \in M(X)$, $u \geqq v$ means $u(x) \geqq v(x)$ for all $x \in X$. For any $p \in P(X)$ and any $u \in M(X)$, $p u$ denotes the integral of $u$ with respect to $p$. The product space of $X$ and $Y$ will be denoted by $X Y$. For any $u \in M(X Y)$ and any $q \in Q(Y \mid X)$, qu denotes the element of $M(X)$ whose value at $x_{0} \in X$ is given by $q u\left(x_{0}\right)=\int_{Y} u\left(x_{0}, y\right) d q\left(y \mid x_{0}\right)$.

A $p \in P(X)$ is degenerate if $p\left\{x_{0}\right\}=1$ for some $x_{0} \subseteq X$, and a $q \in Q(Y \mid X)$ is degenerate if $q(\cdot \mid x)$ is degenerate for each $x$. The degenerate $q$ are exactly those for which there is a Baire function $f$ mapping $X$ into $Y$ such that

$$
q(\{f(x)\} \mid x)=1 \quad \text { for all } x \in X
$$

Any such function $f$ will also denote its associated degenerate $q$, so that, for any $u \in M(X Y)$,

$$
f u(x)=u(x, f(x)) \quad \text { for all } x \in X
$$




\section{$\S 3$. Stopped decision processes.}

In general the optimization problem in our stopped decision processes is defined by six elements $S, A, q, r, g$ and $\alpha$. The state space $S$ and the action space $A$ are non-empty Borel sets, and the law of motion $q$ is a sequence of transition probabilities; $q=\left\{q_{1}, q_{2}, \cdots\right\}$, where each $q_{n} \in Q\left(S \mid H_{n} A\right)$ and $H_{n}=S A \cdots S(2 n-1$ factors $)$ is the set of possible histories of the system when the $n$-th alternative must be chosen. The immediate reward function $r$ is a Baire function on $S A S$, the terminal reward function $g$ is a Baire function on $S$ and $\alpha$ is a discount factor, $0 \leqq \alpha<1$.

But we treat the case only where

$$
r \in M(S A S) \quad \text { and } \quad g \in M(S) .
$$

A policy $\pi$ is a finite sequence $\left\{\pi_{1}, \pi_{2}, \cdots, \pi_{N}\right\}$ or a denumerable sequence $\left\{\pi, \pi_{2}, \cdots\right\}$, where each $\pi_{n} \in Q\left(A \mid H_{n}\right)$. The policy $\pi$ is Markov if each $\pi_{n}$ is a degenerate element of $Q(A \mid S)$, i. e. $\pi=\left\{f_{1}, f_{2}, \cdots\right\}$, where each $f_{n}$ is a Baire function from $S$ into $A$, and stationary if it is Markov and there exists a Baire function $f$ from $S$ into $A$ such that $\pi_{n}=f$ for all $n$.

Any policy $\pi$, together with $q$, defines a conditional probability on the countable product set $A S A S \cdots$ given that the initial state $s$, i. e. it defines

$$
e_{\bar{\pi}}=\pi_{1} q_{1} \pi_{2} q_{2} \cdots \in Q(A S A S \cdots \mid S) .
$$

Let $\subseteq$ and $\mathfrak{i}$ be the Borel fields of $S$ and $A$ respectively. Let the sample space $\Omega=S A S A S \cdots$. The sample point $\omega$ is expressed as $\omega=\left(s_{1}, a_{1}, s_{2}, a_{2}, \cdots\right)$, where each $s_{n} \in S$ and each $a_{n} \in A$. $\mathfrak{F}_{n}$ denotes the family of all unions of sets in $\Omega$ of the form

$$
\left\{\omega \mid s_{1} \in E_{1}, a_{1} \in F_{1}, s_{2} \in E_{2}, a_{2} \in F_{2}, \cdots, s_{n} \in E_{n}\right\}
$$

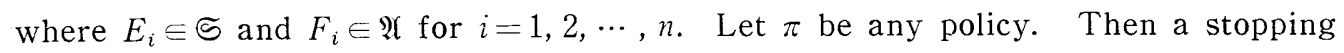
time associated with $\pi$ is a random variable $t=t(\omega)$ with positive integer values such that

and

$$
e_{\pi}\left[\{t(\omega)<\infty\}_{s_{1}} \mid s_{1}\right]=1 \quad \text { for all } s_{1} \in S
$$

$$
\{t(\omega)=n\} \in \mathfrak{F}_{n} \quad \text { for each } n
$$

where by $\{\cdots\}$ we mean the set of all $\omega$ for which the indicated relation holds, and $\{\cdots\}_{s_{1}}$ means the $s_{1}$-section of the $\omega$-set. Let $C(\pi)$, for each $\pi$, be the class of all stopping times associated with $\pi$. For any policy $\pi$ and $t \in C(\pi)$, a pair $(\pi, t)$ is called the stopped-policy (abbreviated as s-policy). $C^{N}(\pi)$ denotes the class of all stopping times $t \in C(\pi)$ for which

$$
e_{\pi}\left[\{t(\omega) \leqq N\}_{s_{1}} \mid s_{1}\right]=1 \quad \text { for all } s_{1} \in S .
$$

An $s$-policy $(\pi, t)$ is called the truncated-policy (abbreviated as $t$-policy) if there exists an integer $N$ such that

$$
t \in C^{N}(\pi) .
$$

Let $\mathfrak{B}_{n}$ denote the family of all unions of sets in $Q$ of the form

$$
\left\{\omega \mid s_{1}, \in E_{1}, s_{2} \in E_{2}, \cdots, s_{n} \in E_{n}\right\}
$$


where $E_{i} \rightleftharpoons \subseteq$ and $\mathscr{G}_{n}$ the family of all $\omega$-sets of the form

$$
\left\{\omega \mid s_{n} \in E_{n}\right\}
$$

where $E_{n} \in \subseteq$. A $t \in C(\pi)$ is Markov if for each $n$

$$
\{t(\omega)=n\} \equiv \mathfrak{B}_{n}
$$

and $\{t(\omega)=n\}$ can be written in the form

$$
\{t(\omega)=n\}=\{t(\omega)>n-1\} \cap \mathcal{S}_{n} \quad \text { for each } n
$$

where $\Delta_{n} \in \mathfrak{G}_{n}$. An $s$-policy $(\pi, t)$ is Markov if both $\pi$ and $t$ are Markov. A Markov $s$-policy is stationary, if $\pi$ is so. A Markov $t \in C(\pi)$ is stationary, if $\Delta_{n}$ does not depend on $n$.

Let

$$
\begin{aligned}
x_{n} & =\sum_{k=1}^{n-1} \alpha^{k-1} r\left(s_{k}, a_{k}, s_{k+1}\right)+\alpha^{n-1} g\left(s_{n}\right) \\
& =\sum_{k=1}^{n-1} \alpha^{k-1} r_{k}+\alpha^{n-1} g_{n} \quad \text { for each } n,
\end{aligned}
$$

where $r_{k}=r\left(s_{k}, a_{k}, s_{k+1}\right)$ and $g_{n}=g\left(s_{n}\right)$.

$E^{-}$denotes the expectation with respect to $e_{-}$. The expected total reward from an $s$-policy $(\pi, t)$, as a function of the initial state $s_{1}$, is then

$$
\begin{aligned}
E^{-}\left(x_{t}\right)\left(s_{1}\right) & =\int_{A S A S .} x_{t} d e_{n}\left(\cdot \mid s_{1}\right) \\
& =\sum_{n=1}^{\infty} \int_{\{t=n\}} x_{n} d\left(\pi_{1} q_{1} \pi_{2} q_{2} \cdots \pi_{n-1} q_{n-1}\right)\left(\cdot \mid s_{1}\right) \\
& =\sum_{n=1}^{\infty} \int_{\{t=n\}}\left[\sum_{k=1}^{n-1} \alpha^{k-1} r_{k}+\alpha^{n-1} g_{n}\right] d\left(\pi_{1} q_{1} \pi_{2} q_{2} \cdots \pi_{n-1} q_{n-1}\right)\left(\cdot \mid s_{1}\right) \\
& =\int_{A S A S \ldots}\left[\sum_{k=1}^{t-1} \alpha^{k-1} r_{k}+\alpha^{t-1} g_{t}\right] d e_{n}\left(\cdot \mid s_{1}\right) .
\end{aligned}
$$

Let $\Pi$ denote the class of all policies and $\Lambda$ the class of all stopped policies, i. e.

$$
A=\{(\pi, t) \mid \pi \in \Pi, t \in C(\pi)\} .
$$

An $s$-policy $\left(\pi^{*}, t^{*}\right)$ is called $\varepsilon$-optimal if

$$
E^{\pi^{*}}\left(x_{t^{*}}\right) \geqq E^{*}\left(x_{t}\right)-\varepsilon \quad \text { for every }(\pi, t) \in A
$$

and is called optimal if

$$
E^{\kappa^{*}}\left(x_{t^{*}}\right) \geqq E^{-*}\left(x_{t}\right) \quad \text { for every }(\pi, t) \in \Lambda .
$$

\section{§4. $\varepsilon$-optimal stationary stopped-policies.}

In this section we shall assume that $A$ is a compact metric space, $r$ a bounded upper semi-continuous function on $S A S, q$ a bounded upper semi-continuous function on $S$ and $q_{1}=q_{2}=\cdots=q \in Q(S \mid S A)$ is continuous, that is, $\left(s_{n}, a_{n}\right) \rightarrow(s, a)$ implies that $q\left(\cdot \mid s_{n}, a_{n}\right)$ converges weakly to $q(\cdot \mid s, a)$. 
We consider the case only where the discount factor $\alpha<1$, and the motion-law of the system is homogeneous Markov We shall use the notations and lemmas closely those of [2].

With any degenerate $f \in Q(A \mid S)$ we associate the operators $T_{i}$ and $A_{f}$, mapping $M(S)$ into $M(S)$ defined by

$$
\begin{aligned}
& T_{f} u(s)=\int[r(s, f(s), \cdot)+\alpha u(\cdot)] d q(\cdot \mid s, f(s)) \\
& A_{f} u(s)=\max \left[g(s), T_{f} u(s)\right]
\end{aligned}
$$

respectively. $T_{f} u(s)$ may be interpretted as the expected return if you are in state $s$, take an action $f(s)$, and receive a final return $u\left(s^{\prime}\right)$ at the resulting state $s^{\prime}$. $A_{r} u(s)$ is the optimal return, when the system allows you to select two possible alternatives in the state $s$ (a) taking an action $f(s)$ or (b) terminating the process. The following properties of the operators $T_{f}, A_{f}$, formulated as a lemma, are immediate from the definition.

LEMMA 4.1. (a) $T_{f}, A_{f}$ are monotone operators.

(b) For any $u \in M(S)$, constant $c, T_{i}(u+c)=T_{f} u+\alpha c$.

(c) For any $u \in M(S)$, constant $c>0, A_{f}(u+c) \leqq A_{f} u+\alpha c$.

PROOF. The proofs are stated in Lemma 7.1 of [2].

Here are some basic properties which will take an important role in proving our desired results.

LEMma 4.2. (a) With any Baire function $f: S \rightarrow A$, the operator $A_{f}$ is a contraction mapping on $M(S)$ with contraction coefficient $\alpha$.

(b) For any $u, v \in M(S)$ and any Markov policy $\pi=\left\{f_{1}, f_{2}, \cdots\right\}$, it holds that

$$
\lim _{n \rightarrow \infty}\left\|A_{f_{1}} A_{f_{2}} \cdots A_{f_{n}} u-A_{f_{1}} A_{f_{2}} \cdots A_{f_{n}} v\right\|=0 .
$$

Proof. (a), (b) are stated in Lemma 7.2 of [2].

We say that a $u \in M(S)$ satisfies the optimality equation, if it holds that

$$
u=\sup _{a:-A} A_{a} u,
$$

where $A_{a}$ is the operator $A_{y}$ with $f \equiv a$. The principal general result on the optimality equation is contained in the following lemma.

LEMMA 4.3. The optimality equation has at most one bounded solution.

Proof. This lemma is stated as Lemma 7.15 in [2].

But under the assumptions stated at the beginning of this section it will be shown that the optimality equation has a unique bounded solution in $C_{1}(S)$.

On the other hand we introduce a process $\left\{\beta_{n}^{N}, n=1,2, \cdots, N\right\}$ for any fixed integer $N \geqq 1$, which is closely related to the operator $A_{f}$. For any policy $\pi$ we define for each $N \geqq 1$ a finite sequence of random variables $\left\{\beta_{n}^{N}(\pi) ; 1 \leqq n \leqq N\right\}$, by backward induction:

$$
\left\{\begin{array}{l}
\beta_{n}^{N}(\pi)=\max \left[x_{n}, \pi_{n} q_{n}\left(\beta_{n-1}^{N}(\pi)\right)\right], \quad n=1,2, \cdots, N-1, \\
\beta_{N}^{N}(\pi)=x_{N} .
\end{array}\right.
$$

We now define the stopping time $\tau_{N}$ 


$$
\tau_{Y}(\pi)=\text { the first integer } n \text { such that } \beta_{n}^{y}(\pi)=x_{n} .
$$

Then it is obvious that

Let

$$
\tau, y(\pi) \equiv C^{y}(\pi)
$$

$$
\alpha^{n-1} \gamma_{n}^{\cdot y}(\pi)=\beta_{n}^{Y}(\pi)-\sum_{k=1}^{n-} \alpha^{k-1} \gamma_{k}
$$

(4.3) then turns out to become

$$
\left\{\begin{array}{l}
v_{n}^{N}(\pi)=\max \left[g_{n}, \pi_{n} q_{n}\left\{\alpha v_{n+1}^{N}(\pi) \div r_{n}\right\}\right], \quad n=1,2, \cdots, N-1, \\
v_{N}^{N}(\pi)=g_{N} .
\end{array}\right.
$$

We shall need the following lemma, in particular, for the stationary policy $\pi=f^{(\infty)}$.

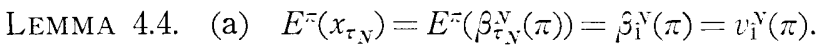

(b) Let $\tau_{N}(\pi)$ be defined by (4.4), then it follows that

$$
\tau_{N}(\pi)=\text { the first integer } n \text { such that } v_{n}^{\mathrm{V}}(\pi)=g_{n} .
$$

(c) For any Markov $\pi=\left\{f_{1}, f_{2}, \cdots\right\}$

$$
A_{f_{1}} A_{f_{2}} \cdots A_{f_{N}} g=v_{1}^{\mathrm{N} \div 1}(\pi) \quad \text { for each } N \geqq 1 .
$$

PRoOF. Lemma 4.5 and 6.2 in [2] show these results.

We have the following lemma, the proof of which is due to [1].

Lemma 4.5. Let $u$ be a bounded upper semi-continuous on $S A$. Define $u^{*}: S \rightarrow R$ by

$$
u^{*}(s)=\max _{a-A} u(s, a) .
$$

Then $u^{*}$ is bounded upper semi-continuous.

Lemma 4.6. Let $w: S \rightarrow R$ be a bounded upper semi-continuous. The $h: S A \rightarrow R$ defined by

$$
h(s, a)=\int w(\cdot) d q(\cdot \mid s, a)
$$

is bounded upper semi-continuous.

Proof. If $w$ is continuous, then clearly $h$ is continuous. Now if $w$ is bounded and upper semi-continuous, there exists a sequence of bounded continuous functions $\left\{w_{n}, n=1,2, \cdots\right\}$ such that

$$
w_{n} \downarrow w \text { on } S .
$$

Let $h_{n}(s, a): S A \rightarrow R$ be defined by

$$
h_{n}(s, a)=\int w_{n}(\cdot) d q(\cdot \mid s, a) .
$$

Each $h_{n}$ is continuous and, moreover, by the dominated convergence theorem,

$$
h_{n} \downarrow h \text { on } S A \text {. }
$$

If $\left(s_{n}, a_{n}\right) \rightarrow(s, a)$, then for any $\varepsilon>0$ there exist integers $N_{1}, N_{2}$ such that

and

$$
n \geqq N_{1} \text { implies } h_{n}(s, a) \leqq h(s, a)+\stackrel{\varepsilon}{3}
$$

$$
m \geqq N_{2} \text { implies }\left|h_{n}(s, a)-h_{n}\left(s_{m}, a_{m}\right)\right| \leqq \begin{aligned}
& \varepsilon \\
& 3
\end{aligned} \quad \text { for } n=1,2, \cdots \text {. }
$$


Therefore

$$
m \geqq N_{2} \text { implies } h_{n}\left(s_{m}, a_{m}\right) \leqq h_{n}(s, a) \div \frac{\varepsilon}{3} \quad \text { for } n=1,2, \cdots .
$$

By (4.5),

$$
h\left(s_{m}, a_{m}\right) \leqq h_{n}\left(s_{m}, a_{m}\right)+\begin{gathered}
\varepsilon \\
3
\end{gathered} \text { for } n=1,2, \cdots .
$$

Combining these results expressed in (4.6), (4.7) and (4.8) yields the desired relation

$$
n \geqq N_{1} \text { and } m \geqq N_{2} \text { imply } h\left(s_{m}, a_{m}\right) \leqq h(s, a)+\varepsilon .
$$

Since $\varepsilon$ is arbitrary we obtain

$$
\limsup _{m \rightarrow \infty} h\left(s_{m}, a_{m}\right) \leqq h(s, a) .
$$

Clearly $h$ is bounded. This completes the proof.

The following theorem due to Dubins and Savage ([3]), was proved in detail by A. Maitra ([1]).

Selection Theorem (A. Maitra [1]): Let $u$ be a bounded upper semi-continuous on $S A$. Then there exists a Baire function from $S$ into $A$ such that

$$
u(s, f(s))=\max _{a=A} u(s, a) \quad \text { for all } s \in S .
$$

For $u, v \in C_{1}(S)$ we define $d_{1}$ by

$$
d_{1}(u, v)=\|u-v\|=\sup _{s: s}|u(s)-v(s)|,
$$

then the following lemma is established by A. Maitra ([1]).

Lemma 4.7. $\left(C_{1}(S), d_{1}\right)$ is a complete metric space.

PROOF. It suffices to show that $C_{1}(S)$ is closed under the uniform convergence. The detailed proof will be omitted here.

Throughout the remainder of this section we will assume that

$$
r=r^{*}+r^{* *}
$$

where $r^{*} \in C_{1}(S A), r^{* *} \in C_{1}(S)$ and $r\left(s, a, s^{\prime}\right)=r^{*}(s, a)+r^{* *}\left(s^{\prime}\right)$. This decomposition of $r$ into $r^{*}$ and $r^{* *}$ means that the reward $r^{* *}\left(s^{\prime}\right)$ related to the new state $s^{\prime}$ is free from the current state $s$ and current action $a$. Of course $r^{*}$ and $r * *$ are not necessarily constant sign. Therefore this restriction on $r$ is rather slight.

For any $u \in C_{1}(S)$, let $A u: S \rightarrow R$ be the function defined by

$$
A u(s)=\max _{a: A} A_{a} u(s) \text {. }
$$

Note that the operator $T_{a}\left(=T_{f}\right.$, where $\left.f \equiv a\right)$, which is the contraction mapping on $M(S)$, maps $C_{1}(S)$ into $C_{1}(S)$, since for $u \in C_{1}(S)$

$$
\begin{aligned}
T_{a} u(s) & =\int_{S}[r(s, a, \cdot)+\alpha u(\cdot)] d q(\cdot \mid s, a) \\
& =\int_{S}[r *(s, a)+r * *(\cdot)+\alpha u(\cdot)] d q(\cdot \mid s, a) \\
& =r *(s, a)+\int_{S}\left[r^{* *}(\cdot)+\alpha u(\cdot)\right] d q(\cdot \mid s, a)
\end{aligned}
$$


implies $T_{a} u \in C_{1}(S)$ for each $a \in A$ because of Lemma 4.6. It is also a contraction mapping on $C_{\mathrm{i}}(S)$ with contraction coefficient $\alpha$ for all $a \in A$. Since for $u, v \in$ $C_{1}(S) \max \{u, v\} \equiv C_{1}(S),(4.2)$ implies that the operator $A_{a}$ is a contraction mapping on $C_{1}(S)$ with contraction coefficient $\alpha$ for all $a \in A$.

For any $u \equiv C_{1}(S)$, from $(4.9)$

$$
\begin{aligned}
A u(s) & =\max _{a} \max \left[g(s), \int_{s}[r(s, a, \cdot)+\alpha u(\cdot)] d q(\cdot \mid s, a)\right] \\
& =\max _{a} \max \left[g(s), \int_{S}\left[r^{*}(s, a)+r^{* *}(\cdot)+\alpha u(\cdot)\right] d q(\cdot \mid s, a)\right] .
\end{aligned}
$$

In this expression, $r * *(\cdot)-\alpha u(\cdot)$ is upper semi-continuous on $S$ and by virture of Lemma 4.6 integral within square bracket on the right-hand side of $(4.9)^{\prime}$ is upper semi-continuous in $(s, a)$. Consequently

$$
\max \left[g(s), \int_{S}[r(s, a, \cdot)-\alpha u(\cdot)] d q(\cdot \mid s, a)\right]
$$

is also upper semi-continuous in $(s, a)$, and maximum is attained for all $s \in S$ because the action space $A$ is compact. Since (4.10) is obviously bounded, Lemma 4.5 implies that $A u$ is upper semi-continuous. Thus the operator $A$ maps $C_{1}(S)$ into $C_{1}(S)$.

Lemma 4.8. The operator $A$ defined by (4.9) is a contraction mapping on $C_{1}(S)$, with contraction coefficient $\alpha$, and consequently, has a unique fixed point.

Proof. Let $u_{1}, u_{2} \in C_{1}(S)$. Clearly $u_{1} \leqq u_{2}+\left\|u_{1}-u_{2}\right\|$. Lemma 4.1 yields

$$
A_{a} u_{1} \leqq A_{a}\left(u_{2}-\left\|u_{1}-u_{2}\right\|\right) \leqq A_{a} u_{2}+\alpha\left\|u_{1}-u_{2}\right\|_{i} \quad \text { for all } a \in A \text {. }
$$

Then

$$
\sup _{a-A} A_{a} u_{1} \leqq \sup _{a-A}\left(A_{a} u_{2}+\alpha\left\|u_{1}-u_{2}\right\|\right)=\sup _{a=A} A_{a} u_{2}+\alpha\left\|u_{1}-u_{2}\right\| \text {. }
$$

In this case 'sup' can be replaced by ' $\max$ ' because of the Lemma 4.5. Therefore

$$
A u_{1}-A u_{2} \leqq \alpha\left\|u_{1}-u_{2}\right\| \text {. }
$$

Interchanging $u_{1}$ and $u_{2}$, we get

$$
A u_{2}-A u_{1} \leqq \alpha\left\|u_{1}-u_{2}\right\| .
$$

Hence, $\left\|A u_{1}-A u_{2}\right\| \leqq \alpha\left\|u_{1}-u_{2}\right\|$, which proves that operator $A$ is a contraction mapping, as $\alpha<1$. Since $C_{1}(S)$ is a complete metric space (Lemma 4.7), it follows from the Banach Fixed Point theorem that the operator $A$ has a unique fixed point in $C_{1}(S)$. This completes the proof.

We set the following assumptions.

(H1) $r=r^{\prime}-r^{\prime \prime}$, where $r^{\prime}$ and $r^{\prime \prime}$ are the real-valued Baire functions on $S A S$ and $r^{\prime \prime} \geqq 0$.

(H2) $E^{-\prime}\left(x_{t}^{\prime}\right)<\infty$ for all $(\pi, t) \in A$, where $x_{n}^{\prime}=\sum_{k=1}^{n-1} \alpha^{k-1} r^{\prime}\left(s_{k}, a_{k}, s_{k+1}\right)+\alpha^{n-1} g\left(s_{n}\right)$.

(H3) $\left\{\left(x_{n}^{\prime}\right)^{-}, n \geqq 1\right\}$ is uniformly integrable w.r.t.e for each $\pi \in \Pi$, where $a^{-}=$ $\max (0,-a)$.

The following lemma is given as Lemma 7.12 in [2].

Lemma 4.9. Let (H1), (H2) and (H3) be satisfied. Any $u \in M(S)$ for which $A_{a} u \leqq u$ 
for all $a \equiv A$ satisfies that $E *\left(x_{i}\right) \leqq u$ for all $(\pi, t) \in A$.

Finally we have the desired result on the basis of above lemmas.

Theorey. Assume (H1), (H2) and (H3). Then for any $\varepsilon>0$ there exists an $\varepsilon$ optimal stationary s-policy which is t-policy.

PROOF. By Lemma (4.8) the operator $A$ has a unique fixed point $u^{*}$ in $C_{1}(S)$, so that

$$
u^{*}=A u^{*}=\max _{a-A} A_{a} u^{*}, \text { where } u^{*} \in C_{1}(S) .
$$

For this $u^{*}$, function $u: S . A \rightarrow R$ defined by

$$
u(s, a)=\max \left[g(s), \int_{s}\left[r(s, a, \cdot) \div \alpha u^{*}(\cdot)\right] d q(\cdot \mid s, a)\right]
$$

is bounded upper semi-continuous on $S A$. From the Selection Theorem there exists a Baire function $f$ from $S$ into $A$ such that

$$
u(s, f(s))=\max _{a: A} u(s, a) \quad \text { for all } \quad s \in S .
$$

Consequently this function defines the operator $A_{f}$ on $M(S)$ such that

$$
u^{*}=A u^{*}=\max _{a \cdot A} A_{a} u^{*}=A_{f} u^{*} .
$$

On the other hand from the Lemma 4.2 the operator $A_{f}$ is a contraction mapping on $M(S)$. Then by virture of Banach Fixed Point theorem, it has a unique fixed point $w^{*}$

$$
A_{f} u^{*}=w^{*} \text {, where } w^{*} \in M(S) .
$$

The uniqueness of the fixed point of $A_{j}$ together with the fact $C_{1}(S) \leqq M(S)$ yields

$$
u^{*}=w^{*} .
$$

Hence $A_{f} u^{*}=u^{*}$, so that $\left(A_{f}\right)^{n} u^{*}=u^{*}$ for all $n \geqq 1$. From Lemma 4.2 , for any $\varepsilon>0$ there exists an integer $N$ such that $n \geqq N$ implies

$$
\left(A_{f}\right)^{n-1} g \geqq\left(A_{f}\right)^{n-1} u *-\varepsilon=u^{*}-\varepsilon .
$$

Since $u^{*}$ satisfies the optimality equation, $u^{*} \geqq A_{a} u^{*}$ for all $a \in A$.

Then by Lemma 4.9

$$
u^{*} \geqq E^{-}\left(x_{t}\right) \quad \text { for all }(\pi, t) \in A .
$$

Combining the results expressed in (4.12) and (4.13) yields the relation

$$
\left(A_{t}\right)^{n-1} g \geqq E^{-*}\left(x_{t}\right)-\varepsilon \text { for any }(\pi, t) \in A \text {, where } n \geqq N \text {. }
$$

Lemma 4.4 allows us to write

where $f^{\prime}=(f, f, \cdots)$ and

$$
E^{f^{(\infty)}}\left(x_{\tau_{n}(f)}\right)=\left(A_{f}\right)^{n-1} g
$$

$$
\tau_{n}(f)=\text { the first integer } k \text { such that } v_{k}^{n}\left(f^{(\infty)}\right)=g_{k} .
$$

Finally, using (4.14) and (4.15) we obtain the final relation

$$
E^{f^{(\infty)}}\left(x_{\tau_{n}(f)}\right) \geqq E^{\bar{\sigma}}\left(x_{t}\right)-\varepsilon \text { for all }(\pi, t) \in \Lambda \text { and } n \geqq N .
$$


It is obvious that $s$-policy $\left(f^{\infty}, \tau_{n}(f)\right)$ is truncated, i. e.

$$
\left(f^{\infty \infty}, \tau_{n}(f)\right) \in C^{n}\left(f^{n<\infty}\right)
$$

and that it is $\varepsilon$-optimal stationary s-policy for $n \geqq N$. This completes the proof.

Moreover if we put the following assumptions in addition to (H1) and (H3) we can find an optimal s-policy which has a stationary stopping time.

(H4) $\lim _{n \rightarrow \infty} x_{n}^{\prime \prime}=+\infty u^{\prime} e_{-.} .1$ for all $\pi \in I I$, where $x_{n}^{\prime \prime}=\sum_{k=1}^{n-1} \alpha^{k-1} r^{\prime \prime}\left(s_{k}, a_{k}, s_{k \div 1}\right)$.

(H5) (i) $\left\{\left(x_{n}^{\prime}\right)^{-}, n \geq 1\right\}$ is uniformly integrable w.r.t.e $e_{-,}$, and (ii) $\sup _{v} E^{f^{(\infty)}}\left(x_{N}^{\prime} f\right)<\infty$ for every stationary $f^{(\infty)}$.

Corollary. Assume (H1), (H2), (H4) and (H5). Then there exists an optimal stationary s-policy having a stationary stopping time.

PROOF.

$$
\lim _{n \rightarrow \infty}\left(A_{f}\right)^{n} g=v_{1}\left(f^{(\infty)}\right)=u^{*}, \quad v_{n}\left(f^{(\infty)}\right)=\lim _{x \rightarrow \infty} v_{n}^{N}\left(f^{(\infty)}\right)
$$

and $\tau$ be the first $n$ such that $v_{n}\left(f^{(\infty)}\right)=g_{n}$.

The arguments in [2], Corollary 7.2, Theorem 8.2, show us the facts

$$
\tau \in C\left(f^{(\infty)}\right)
$$

and

$$
\sup _{i \in C(f(\infty))} E^{f^{(\infty)}}\left(x_{t}\right)=\lim _{x \rightarrow \infty} E^{f^{(\infty)}}\left(x_{\tau_{X}(i)}\right),
$$

which completes the proof.

In conclusion we remark that in paper $[1]$, even if $r(s, a)$ is replaced by $r\left(s, a, s^{\prime}\right)$ $=r^{*}(s, a)_{\top} r^{* *}\left(s^{\prime}\right)$, where $r^{*}(s, a) \in C_{1}(S A), r^{* *}\left(s^{\prime}\right) \in C_{1}(S)$, the Maitra's result remains true: there exists a stationary plan.

Acknowledgement. I would like to thank Dr. N. Furukawa, under whose guidance this paper was prepared.

\section{References}

[1] A. MAITRA, Discounted dynamic programming on compact metric space, Sankhyā. series A. vol 30, (1968), pp. 211-216.

2 N. Furlkawa and S. Iwamoto, Stopped Decision Processes on Complete Separable Metric Spaces. to appear. J. Math. Anal. Appl.

$3]$ L.E. Dlbixs and L. J. SAvage, How to Gamble If You Must. MacGraw.Hill, New York (1965). 